\title{
MULTIPATH DETECTION OF STOCHASTIC TRANSIENT PROCESSES
}

\author{
Francisco M. Garcia and Isabel M. G. Lourtie \\ ISR -Instituto de Sistemas e Robótica, IST - Instituto Superior Técnico \\ Torre Norte, Av. Rovisco Pais, 1049-001 Lisboa, Portugal \\ E-mail : fmg@isr.ist.utl.pt
}

\begin{abstract}
This paper reports on the detection of Gaussian stochastic transients in multipath environments described by random parameters. The solutions developed herein correspond to quadratic processors, with low computational cost and robust to changes in the statistical models of the channel. As a consequence, only a small amount of a-priori information is necessary to derive the parameters of the processor. A recursive form of the processor is also proposed, allowing for the recursive detection of the signal replicas arriving at the receiver.
\end{abstract}

\section{INTRODUCTION}

The classical solution for the detection of signals in a multipath environment is the generalized likelihood ratio test (GLRT), where the likelihood test is computed from estimates of the channel parameters in both hypotheses. In general, the estimation step in the GLRT is a heavy computational procedure. Furthermore, when the signals to detect are stochastic processes in low signal-to-noise ratio (SNR), which is the usual case in many passive detection applications, the variances of the estimates are large and the detector performance degrades. Recently, some authors have developed suboptimal processors to avoid the drawbacks of the GLRT. In [1], a suboptimal approximation for the detection of continuoustime, stationary processes in low SNR was proposed, assuming that the channel parameters are random variables. This processor was based on a Taylor series approximation of the likelihood ratio for the processor with known channel parameters. In [2], a geometric framework based on multiresolution techniques was proposed, where the set of all possible signals arriving at the receiver is approximated by a simpler linear subspace. The detectors proposed in [1] and [2] are both quadratic processors.

This paper extends the work of [1] to short-duration stochastic transients, which are nonstationary in nature. The following situation is considered: i) the processor is developed in discrete time; ii) the multipath channel is regarded as a "signal amplifier", instead of a nuisance; iii) the low SNR condition assumes that the signal eigenvalues are smaller than the noise ones. The solutions proposed have two major concerns. First, they must rely on a small a-priori amount of statistical information about the channel parameters. The basic idea is that this information should be easily inferred from local data (i.e., depth, salinity, temperature in an underwater media) and, when the local conditions change, the processor parameters must be recalculated fast. The simulation results show that the processors are robust to mismatches on the channel parameters, and thus only mild information about the range of the delay coefficients and approximate estimates of the attenuation coefficient means and variances are necessary. Second, the computational cost of the resulting processors must be low, allowing for real-time processing. Two possible processing structures are proposed: i) a quadratic form, which can be directly optimized in terms of a performance/computational complexity compromise using the methods proposed in [3]; ii) a recursive solution, where a sequence of tests are performed at the arrival of each signal replica. In most situations, this scheme reduces both the computational cost of the processor, and the mean time interval between the arrival of the first replica at the receiver and its detection time instant.

\section{PROBLEM FORMULATION}

The detection problem is formulated as a simple binary test. The channel is modeled such that the signal arriving at the receiver under hypothesis $H_{1}$ is a weighted sum of delayed replicas of the emitted signal. Thus, the observation process $r(t)$ is defined as

$$
r(t)= \begin{cases}y(t)+n(t), & \text { under hypothesis } H_{1} \\ n(t), & \text { under hypothesis } H_{0},\end{cases}
$$

where

$$
\begin{gathered}
y(t)=\alpha_{1} s\left(t-T_{0}\right)+\sum_{k=2}^{N_{q}} \alpha_{k} s\left(t-T_{0}-\tau_{k}\right) \\
\Leftrightarrow \quad y\left(t+T_{0}\right)=\sum_{k=1}^{N_{q}} \alpha_{k} s\left(t-\tau_{k}\right), \quad \text { with } \tau_{1}=0 .
\end{gathered}
$$

In (2), $T_{0}, \alpha_{k}$ and $\tau_{k}, k=1, \cdots, N_{q}$ denote, respectively, the time delay between the emission and the reception of the first replica of a signal $s(t)$, the attenuation coefficients (AC) and the delay coefficients (DC), where $N_{q}$ represents the number of replicas arriving at the receiver. The emitted signal, $s(t)$, is a Gaussian, zeromean transient with autocorrelation function $k_{s}\left(t_{1}, t_{2}\right)$. The noise, $n(t)$, is stationary, Gaussian distributed with zero-mean and with constant power spectrum up to a high frequency. The observation process is conveniently filtered and discretized at a sampling frequency $T_{s}$. For simplicity, we assume in the sequel that the sampled noise sequence is white, and that the DCs, $\tau_{k}$, are integer multiples of $T_{s}$, i.e., $\tau_{k}=q_{k} T_{s}, k=1, \cdots, N_{q}$. The optimal detector in this case corresponds to the likelihood test

$$
\frac{E_{\boldsymbol{q}, \boldsymbol{\alpha}}\left[p_{H_{1}}\left(\boldsymbol{r} \mid H_{1}, \boldsymbol{q}, \boldsymbol{\alpha}\right)\right]}{E_{\boldsymbol{q}, \boldsymbol{\alpha}}\left[p_{H_{0}}\left(\boldsymbol{r} \mid H_{0}, \boldsymbol{q}, \boldsymbol{\alpha}\right)\right]}=E_{\boldsymbol{q}, \boldsymbol{\alpha}}\left[\frac{p_{H_{1}}\left(\boldsymbol{r} \mid H_{1}, \boldsymbol{q}, \boldsymbol{\alpha}\right)}{p_{H_{0}}\left(\boldsymbol{r} \mid H_{0}\right)}\right] \underset{H_{0}}{\stackrel{H_{1}}{>}} \eta,
$$


where $\boldsymbol{q}=\left\{q_{1}, \cdots, q_{N_{q}}\right\}, \boldsymbol{\alpha}=\left\{\alpha_{1}, \cdots, \alpha_{N_{q}}\right\}, \boldsymbol{r}=\left[r\left(T_{s}+\right.\right.$ $\left.\left.T_{0}\right) \cdots r\left(N T_{s}+T_{0}\right)\right]^{\prime}$ corresponds to the observation vector over an interval of length $N$ and $p_{H_{i}}\left(\boldsymbol{r} \mid H_{i}, \boldsymbol{q}, \boldsymbol{\alpha}\right)$ represents the probability density function of $\boldsymbol{r}$ given $\boldsymbol{q}$ and $\boldsymbol{\alpha}$, under hypothesis $H_{i}$, $i=0,1$. It is assumed that the interval $N$ is large enough to include all the replicas of an emitted signal arriving at the receiver.

\section{LIKELIHOOD RATIO FOR KNOWN AC AND DC}

Since $y(t)$ consists on a sum of zero-mean Gaussian-distributed signals, then the joint probability density function of $\boldsymbol{r}$ under both hypothesis is also Gaussian. Thus, the term inside brackets in (3) may be rewritten as

$l(\boldsymbol{r})=\frac{p_{H_{1}}\left(\boldsymbol{r} \mid H_{1}, \boldsymbol{q}, \boldsymbol{\alpha}\right)}{p_{H_{0}}\left(\boldsymbol{r} \mid H_{0}\right)}=\sqrt{\frac{\left|\boldsymbol{C}_{H_{0}}\right|}{\left|\boldsymbol{C}_{H_{1}}\right|}} \exp \left(-\frac{1}{2} \boldsymbol{r}^{\prime}\left[\boldsymbol{C}_{H_{1}}^{-1}-\boldsymbol{C}_{H_{0}}^{-1}\right] \boldsymbol{r}\right)$,

where $C_{H_{i}}$ corresponds to the covariance matrix of dimension $(N \times N)$ of $\boldsymbol{r}$ under hypothesis $H_{i}$ and $|\cdot|$ denotes the determinant. Let $\sigma^{2}$ be the variance of the discretized noise and $\boldsymbol{C}_{y}$ the covariance matrix of the discrete received signal, $\boldsymbol{y}$, under hypothesis $H_{1}$. Then, $\boldsymbol{C}_{H_{0}}=\sigma^{2} \boldsymbol{I}$ and $\boldsymbol{C}_{H_{1}}=\sigma^{2} \boldsymbol{I}+\boldsymbol{C}_{y}$ (I represents the identity matrix). Denote by $\boldsymbol{C}_{s}$ the $\left(N_{1} \times N_{1}\right)$ covariance matrix of the discretized emitted transient signal $s(t)$, where $N_{1}$ represents the interval where most of the signal energy lies. Consider the decomposition $\boldsymbol{C}_{s}=\boldsymbol{V}_{s} \boldsymbol{D} \boldsymbol{V}_{s}^{\prime}$, where $\boldsymbol{V}_{s}\left(N_{1} \times N_{\lambda}\right)$ and $\boldsymbol{D}=\operatorname{diag}\left\{\lambda_{1}^{s}, \cdots, \lambda_{N_{\lambda}}\right\}$ are, respectively, the eigenvector and eigenvalue matrices of $\boldsymbol{C}_{s}$. Under these conditions, we have

$$
C_{y}=V_{y} D V_{y}^{\prime}
$$

with

$$
\boldsymbol{V}_{y}=\sum_{k=1}^{N_{q}} \alpha_{k} \boldsymbol{V}_{s}^{q_{k}}
$$

and

$$
\boldsymbol{V}_{s}^{k}=\left[\begin{array}{c}
\boldsymbol{o}\left(k, N_{\lambda}\right) \\
\boldsymbol{V}_{s} \\
\boldsymbol{o}\left(N-N_{1}-k, N_{\lambda}\right)
\end{array}\right]
$$

where $\boldsymbol{o}(n, m)$ stands for the $(n \times m)$ null matrix. It is easy to show [4] that the likelihood ratio (4) can be rewritten as

$$
l(\boldsymbol{r})=\exp \left[\frac{1}{2} \ln \left(\left|\boldsymbol{I}-\sigma^{2} \boldsymbol{V}_{y}^{\prime} \boldsymbol{V}_{y} \boldsymbol{W}_{y}\right|\right)+\frac{1}{2} \boldsymbol{r}^{\prime} \boldsymbol{V}_{y} \boldsymbol{W}_{y} \boldsymbol{V}_{y}^{\prime} \boldsymbol{r}\right],
$$

with

$$
\begin{aligned}
\boldsymbol{W}_{y} & =\boldsymbol{D}_{2}\left(\boldsymbol{U}_{1} \boldsymbol{D}_{2}+\boldsymbol{I}\right)^{-1} / \sigma^{2} \\
\boldsymbol{U}_{1} & =\sum_{k=1}^{N_{q}} \sum_{\substack{l=1 \\
l \neq k}}^{N_{q}} \alpha_{k} \alpha_{l}\left(\boldsymbol{V}_{s}^{k}\right)^{\prime} \boldsymbol{V}_{s}^{l} \\
\boldsymbol{D}_{2} & =\left(c_{1} \boldsymbol{I}+\sigma^{2} \boldsymbol{D}^{-1}\right)^{-1},
\end{aligned}
$$

where $c_{1}=\sum_{k=1}^{N_{q}} \alpha_{k}^{2}$.

Remark that the optimal detector corresponds to taking the expected value in order to the ACs and DCs of (8). However, the resulting processor has no closed-form expression and requires a huge computational load. To overcome this inconvenient, we simplify (8) by taking the terms up to the first order of its Taylor series around a convenient working point. Under the conditions that the
SNR is low (i.e., $\lambda_{k}<\sigma^{2}, \forall k=1, \cdots, N_{\lambda}$ ) and the multipath channel amplifies the signal energy arriving at the receiver (i.e., $c_{1}>1$ ), then the elements of the diagonal of the matrix $D_{2}$ are small. Thus, the first-order approximation of the likelihood ratio around the point $D_{2}=\boldsymbol{o}\left(N_{\lambda}, N_{\lambda}\right)$ is

$$
l(\boldsymbol{r}) \simeq 1-\frac{1}{2} \operatorname{tr}\left\{\boldsymbol{V}_{y} \boldsymbol{D}_{2} \boldsymbol{V}_{y}^{\prime}\right\}+\frac{1}{2 \sigma^{2}} \boldsymbol{r}^{\prime} \boldsymbol{V}_{y} \boldsymbol{D}_{2} \boldsymbol{V}_{y}^{\prime} \boldsymbol{r}=\pi(\boldsymbol{r}) .
$$

When the DCs and the ACs are known, $\pi(\boldsymbol{r})$ still represents the optimum solution when there is no overlapping between the replicas arriving at the receiver, since $\boldsymbol{U}_{1}=\boldsymbol{o}\left(N_{\lambda}, N_{\lambda}\right)$. This situation corresponds to the case where the duration of the emitted signal is small comparing to the channel delays. However, the examples presented in [4] show that, even when there is a large overlapping between replicas, the power of the signal arriving at the receiver increases and the performance of the processor does not degrade significantly.

In the next section the suboptimal processor is derived by taking the expected value in order to the ACs, $\alpha$, and DCs, $\boldsymbol{q}$, of the approximated likelihood ratio $\pi(\boldsymbol{r})$ (12).

\section{EXPECTED VALUE OF $\pi(\boldsymbol{r})$}

In the sequel, the elements of $\left\{\alpha_{1}, \cdots, \alpha_{N_{q}}, q_{1}, \cdots, q_{N_{q}}\right\}$ are assumed to be mutually independent. By taking the expected value in order to the ACs, $\boldsymbol{\alpha}$, one gets

$$
E_{\boldsymbol{\alpha}}[\pi(\boldsymbol{r})]=1+\frac{1}{2 \sigma^{2}} \boldsymbol{r}^{\prime} E_{\boldsymbol{\alpha}}\left[\boldsymbol{V}_{y} \boldsymbol{D}_{2} \boldsymbol{V}_{y}^{\prime}\right] \boldsymbol{r}-\frac{1}{2} \operatorname{tr}\left\{E_{\boldsymbol{\alpha}}\left[\boldsymbol{V}_{y} \boldsymbol{D}_{2} \boldsymbol{V}_{y}^{\prime}\right]\right\},
$$

where $\operatorname{tr}\{\boldsymbol{X}\}$ denotes the trace of $\boldsymbol{X}$. Since $\boldsymbol{D}_{2}$ depends on $\boldsymbol{\alpha}$ through $c_{1}$, the expected value in (13) should be evaluated using the joint probability density function of $\boldsymbol{\alpha}$. However, if we assume that $c_{1}=c_{1}^{c}$ is approximately constant, it is only necessary to know the first and second order statistics of $\boldsymbol{\alpha}$, i.e.,

$$
E_{\boldsymbol{\alpha}}\left[\boldsymbol{V}_{y} \boldsymbol{D}_{2} \boldsymbol{V}_{y}^{\prime}\right] \simeq \sum_{k_{1}=1}^{N_{q}} \sum_{k_{2}=1}^{N_{q}} C_{\alpha_{k_{1}} \alpha_{k_{2}}} \boldsymbol{V}_{s}^{q_{k_{1}}} \boldsymbol{D}_{2}\left(\boldsymbol{V}_{s}^{q_{k_{2}}}\right)^{\prime},
$$

where $C_{\alpha_{k_{1}} \alpha_{k_{2}}}=E\left[\alpha_{k_{1}} \alpha_{k_{2}}\right]$ represents the crosscorrelation between $\alpha_{k_{1}}$ and $\alpha_{k_{2}}$. The constant $c_{1}^{c}$ is for now left as a free parameter that will be chosen in order to maximize the performance of the final expression of the processor.

The final expression of the likelihood test is obtained taking the expected value of (13)with respect to the DCs, $\boldsymbol{q}$, i.e.,

$$
\pi_{p}(\boldsymbol{r}) \stackrel{H_{1}}{\underset{H_{0}}{\gtrless}} \mu
$$

where the threshold $\mu$ includes the terms of (13) that do not depend on the observation process $r$, and

$\pi_{p}(\boldsymbol{r})=\boldsymbol{r}^{\prime}\left\{\sum_{k_{1}=1}^{N_{q}} \sum_{k_{2}=1}^{N_{q}} C_{\alpha_{k_{1}} \alpha_{k_{2}}} E_{q_{k_{1}}, q_{k_{2}}}\left[\boldsymbol{V}_{s}^{q_{k_{1}}} \boldsymbol{D}_{2}\left(\boldsymbol{V}_{s}^{q_{k_{2}}}\right)^{\prime}\right]\right\} \boldsymbol{r}$.

When $k=k_{1}=k_{2}$ we have

$$
\overline{\boldsymbol{C}}_{k}=E_{q_{k}}\left[\boldsymbol{V}_{s}^{q_{k}} \boldsymbol{D}_{2}\left(\boldsymbol{V}_{s}^{q_{k}}\right)^{\prime}\right]=\sum_{m=1}^{N_{q}} \boldsymbol{V}_{s}^{m} \boldsymbol{D}_{2}\left(\boldsymbol{V}_{s}^{m}\right)^{\prime} P_{k}(m),
$$


where $P_{k}(m)$ denotes the probability function of $q_{k}$, and for $k_{1} \neq$ $k_{2}$,

$$
E_{q_{k_{1}}, q_{k_{2}}}\left[\boldsymbol{V}_{s}^{q_{k_{1}}} \boldsymbol{D}_{2}\left(\boldsymbol{V}_{s}^{q_{k_{2}}}\right)^{\prime}\right]=\overline{\boldsymbol{V}}_{k_{1}} \boldsymbol{D}_{2} \overline{\boldsymbol{V}}_{k_{2}}^{\prime}
$$

with

$$
\overline{\boldsymbol{V}}_{k}=\sum_{m=1}^{N_{q}} \boldsymbol{V}_{s}^{m} P_{k}(m) .
$$

The probability $P_{k}(m)$ related to the DC $q_{k}$, corresponds to an uncertainty measure of the time interval between the arrival of the first and the $k$-th replica at the receiver. Thus, as noted before, $q_{1}=0$, and $P_{1}(m)=\delta_{m}$ (the kronecker delta). The quadratic form of the likelihood ratio is given by

$\pi_{p}(\boldsymbol{r})=\boldsymbol{r}^{\prime}\left\{\sum_{k=1}^{N_{q}} C_{\alpha_{k}} \overline{\boldsymbol{C}}_{k}+\sum_{k_{1}=1}^{N_{q}} \sum_{\substack{k_{2}=1 \\ k_{1} \neq k_{2}}}^{N_{q}} \bar{\alpha}_{k_{1}} \bar{\alpha}_{k_{2}} \overline{\boldsymbol{V}}_{k_{1}} \boldsymbol{D}_{2} \overline{\boldsymbol{V}}_{k_{2}}^{\prime}\right\} \boldsymbol{r}$

where $C_{\alpha_{k}}=C_{\alpha_{k} \alpha_{k}}=\bar{\alpha}_{k}^{2}+\sigma_{k}^{2}\left(\bar{\alpha}_{k}\right.$ and $\sigma_{k}^{2}$ being, respectively, the mean and variance of $\alpha_{k}$ ).

In (20), matrices $\overline{\boldsymbol{C}}_{k}$ and $\boldsymbol{D}_{2}$ depend on the constant $c_{1}^{c}$, that is tuned in order to maximize the detector performance, using the following procedure: i) for each possible value of $c_{1}^{c}$, let $M$ be the matrix inside brackets in (20), and determine the covariance matrix $C_{H_{1}}^{*}$ that corresponds to a hypothesis for which the processor (20) is optimal, i.e., $\boldsymbol{M}=\boldsymbol{C}_{H_{0}}^{-1}-\left(\boldsymbol{C}_{H_{1}}^{*}\right)^{-1}\left(\boldsymbol{C}_{H_{0}}=\sigma^{2} \boldsymbol{I}\right)$; ii) choose the value of $c_{1}^{c}$ that maximizes either the Chernoff or the Bhattacharyya distances [5] between $C_{H_{1}}^{*}$ and $C_{H_{0}}$. This procedure corresponds to minimizing a bound for the probability of error between both hypotheses and is computationally efficient.

The processor presented in (20) is a quadratic form. Its computational cost is relatively low, comparing with the GLRT, because the matrix $M$ can be computed off-line when the conditions of the multipath channel change. Although the processor depends on the probability functions of the delay coefficients which may not be easy to determine, the simulation studies show that the receiver performance is robust to mismatches on these probabilities. Thus, when the true $P_{k}(m)$ are unknown, the processor is designed assuming uniform probabilities and, in general, the resulting performance degradation is not important.

\section{SEQUENTIAL DETECTION OF REPLICAS}

In (20), the length $N$ of the observation vector $\boldsymbol{r}$ must be large enough to include all replicas arriving at the receiver. Therefore, it is only possible to detect a signal when its last replica arrives. This section develops a sequential structure based on (20) that allows the detection of a signal without the need of waiting for all replicas. In this case, the detection is performed as soon as an arbitrary number of replicas possess a sufficient amount of energy to ensure with high probability that a signal was emitted by the source. Furthermore, in many situations, the sequential detection of replicas may reduce the computational cost of the processor.

In (20), matrices $\overline{\boldsymbol{C}}_{k}$ and $\overline{\boldsymbol{V}}_{k}$ have dimensions $(N \times N)$ and $\left(N \times N_{\lambda}\right)$. However, the dimension of the non-null terms of each of these matrices is significantly lower than $N$ due either to the transient characteristic of the emitted signal and also because it is assumed that the probabilities $P_{k}(m)$ associated to each replica have compact support. Let $\left[\theta_{k}^{i} ; \theta_{k}^{f}\right]$ be the support of $P_{k}(m)$ (with $\left.\theta_{1}^{i}=0\right)$; then, the matrices $\overline{\boldsymbol{C}}_{k}$ and $\overline{\boldsymbol{V}}_{k}$ have, respectively, support $\left[\theta_{k}^{i}+1 ; \theta_{k}^{f}+N_{1}\right] \times\left[\theta_{k}^{i}+1 ; \theta_{k}^{f}+N_{1}\right]$ and $\left[\theta_{k}^{i}+1 ; \theta_{k}^{f}+N_{1}\right] \times\left[1 ; N_{\lambda}\right]$. Defining $N_{q_{k}}=\theta_{k}^{f}-\theta_{k}^{i}+N_{1}$, we denote by $\overline{\boldsymbol{C}}_{k}^{r}\left(N_{q_{k}} \times N_{q_{k}}\right)$ and $\overline{\boldsymbol{V}}_{k}^{r}\left(N_{q_{k}} \times N_{\lambda}\right)$ the matrices that include only the elements belonging to the support of $\overline{\boldsymbol{C}}_{k}$ and $\overline{\boldsymbol{V}}_{k}$. If, at each time instant $n$, the vector of the last $N_{q_{k}}$ elements of the observation process is represented by $\boldsymbol{r}_{k}(n)=\left[r\left(n-N_{q_{k}}+1\right) \cdots r(n)\right]^{\prime}$, then expression (20) may be rewritten as

$$
\begin{aligned}
& \pi_{p}(\boldsymbol{r})=\sum_{k=1}^{N_{q}}\left(\bar{\alpha}_{k}^{2}+\sigma_{k}^{2}\right) l_{k}\left(\theta_{k}^{f}+N_{1}\right) \\
& +2 \sum_{k_{1}=2}^{N_{q}} \sum_{k_{2}=1}^{k_{1}-1} \bar{\alpha}_{k_{1}} \bar{\alpha}_{k_{2}} \boldsymbol{b}_{k_{1}}^{\prime}\left(\theta_{k_{1}}^{f}+N_{1}\right) \boldsymbol{D}_{2} \boldsymbol{b}_{k_{2}}\left(\theta_{k_{2}}^{f}+N_{1}\right),
\end{aligned}
$$

where

$$
l_{k}(n)=\boldsymbol{r}_{k}^{\prime}(n) \boldsymbol{Z}_{k} \boldsymbol{D}_{k}^{z} \boldsymbol{Z}_{k}^{\prime} \boldsymbol{r}_{k}(n) \quad \text { and } \quad \boldsymbol{b}_{k}(n)=\left(\overline{\boldsymbol{V}}_{k}^{r}\right)^{\prime} \boldsymbol{r}_{k}(n) .
$$

In (22), $\boldsymbol{Z}_{k}$ and $\boldsymbol{D}_{k}^{z}$ represent, respectively, the matrices of eigenvectors and eigenvalues of $\overline{\boldsymbol{C}}_{k}^{r}\left(\overline{\boldsymbol{C}}_{k}^{r}=\boldsymbol{Z}_{k} \boldsymbol{D}_{k}^{z} \boldsymbol{Z}_{k}^{\prime}\right)$. Remark that, due to the fact that $q_{1}=0$ and $P_{1}(m)=\delta_{m}$, then $\boldsymbol{Z}_{1}=\boldsymbol{V}_{s}$ and $\boldsymbol{D}_{1}^{z}=\boldsymbol{D}_{2}$. Under these conditions, the likelihood ratio may be rewritten in a recursive form:

$$
\begin{aligned}
\pi_{h}(n) & =\pi_{h-1}\left(n-\theta_{h}^{f}+\theta_{h-1}^{f}\right)+\left(\bar{\alpha}_{h}^{2}+\sigma_{h}^{2}\right) l_{h}(n) \\
& +2 \bar{\alpha}_{h}\left[\sum_{k=1}^{h-1} \bar{\alpha}_{k} \boldsymbol{b}_{k}^{\prime}\left(n-\theta_{h}^{f}+\theta_{k}^{f}\right)\right] \boldsymbol{D}_{2} \boldsymbol{b}_{h}(n),(23)
\end{aligned}
$$

with $\pi_{1}(n)=\left(\bar{\alpha}_{1}^{2}+\sigma^{2}\right) l_{1}(n)$. For $h=N_{q}, \pi_{N_{q}}(n)$ corresponds to expression (20).

Comparing expressions (20) and (23) from a computational point of view, the solution that leads to a less expensive processor depends both on the signals to detect and on the multipath channel structure. In general, however, two situations may arise. First, when there is a large overlapping between replicas, using the recursive processor (23), it is necessary to perform $N_{q}$ eigenvector decompositions of length $N_{q_{k}}$, while the non-recursive form (20) only needs one eigenvector decomposition of length $N$. Since, in this case, $N \ll \sum_{k} N_{q_{k}}$, we should expect that the non-recursive solution would be less expensive than the recursive one. However, this may not be true because, in most cases, the number of relevant eigenvalues is much more important in the non-recursive processor, thus increasing its computational cost. When the overlapping is smaller, then clearly the recursive solution becomes more attractive than the non-recursive one.

From the recursive processor (23), it is possible to derive a detection structure suited for sequential detection of replicas. For every $h=1, \cdots, N_{q-1}$, two likelihood tests are performed. The first test compares $\pi_{h}(n)$ with a high-valued threshold, $\mu_{h}^{\text {sup }}$; if $\pi_{h}(n)>\mu_{h}^{s u p}$, then it is assumed that a signal arrived to the receiver with a low probability of false alarm, and there is no need to evaluate $\pi_{h+1}\left(n+\theta_{h+1}^{f}-\theta_{h}^{f}\right)$, that corresponds to the arrival of the next replica; if $\pi_{h}(n)<\mu_{h}^{\text {sup }}$, then another test is performed against a low threshold $\mu_{h}^{i n f}$; if $\pi_{h}(n)<\mu_{h}^{i n f}$, we consider that no signal is present at the receiver (with low miss probability) and the procedure stops. Only in the case where $\mu_{h}^{i n f} \leq \pi_{h}(n) \leq \mu_{h}^{\text {sup }}$, the processor waits for the next replica to make a decision. When $h=N_{q}$, only one final test is performed. 


\section{SIMULATION RESULTS}

The received signal is a weighted sum of 10 delayed replicas of a chirplike stochastic transient with autocorrelation function shown in figure 1 . The noise variance is $\sigma^{2}=5$. The mean value for the overlapping between consecutive replicas $\bar{\Delta}_{q}$, is $80 \%$ or $20 \%$.

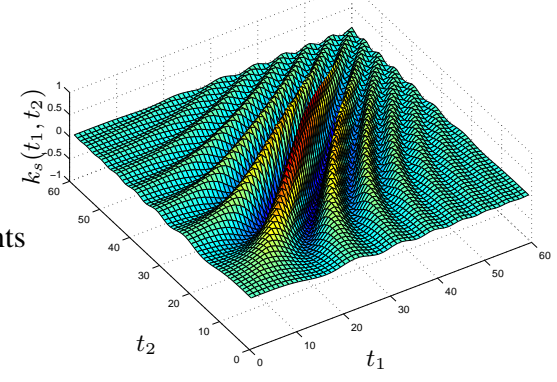

Fig. 1. Autocorrelation function of the emitted signal.
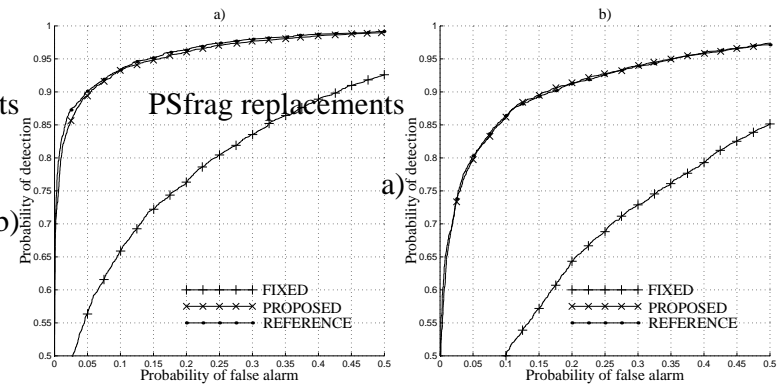

Fig. 2. ROCs: a) $\bar{\Delta}_{q}=80 \%$. b) $\bar{\Delta}_{q}=20 \%$.
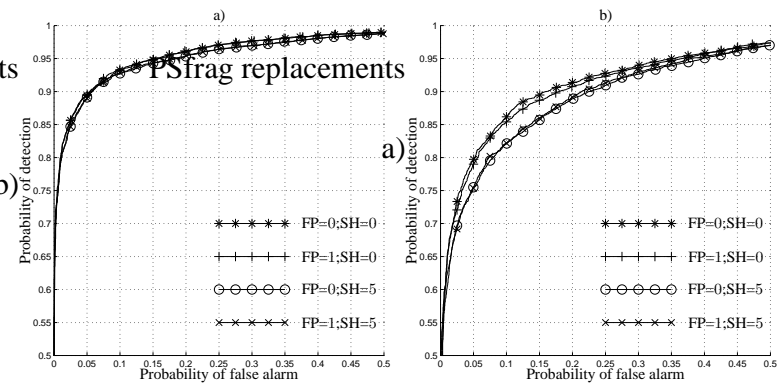

Fig. 3. Statistics mismatch: a) $\bar{\Delta}_{q}=80 \%$. b) $\bar{\Delta}_{q}=20 \%$.

The DCs are generated from a Gaussian probability function with $1 / 3$ rd of the length of the emitted signal, while the ACs have $\bar{\alpha}_{k}=1$ and $\sigma_{k}=0.2, \forall k=1, \cdots, 10$. The receiver operating characteristics (ROC) are obtained by simulation with 5000 Monte-Carlo runs. In figures 2 a) and b), the performance of the proposed detector (PROPOSED) is compared with i) a reference one (REFERENCE), consisting on the best possible quadratic processor obtained from the covariance matrix estimated from the 5000 signals arriving at the receiver; and ii) a detector that assumes that the DCs and ACs take fixed values (FIXED), equal to the mean values of the real channel parameters. We conclude that, for both overlapping situations, the performance of the proposed solution is very close to the best quadratic processor and presents
Table 1. Sequential detection of replicas

\begin{tabular}{|c|c|c|c|}
\hline Scenario & PFA & PD & CC \\
\hline i) & 0.1810 & 0.7102 & $18.16 \%$ \\
\hline ii) & 0.1064 & 0.8374 & $65.31 \%$ \\
\hline iii) & 0.1246 & 0.8140 & $41.59 \%$ \\
\hline
\end{tabular}

a huge gain comparing to the fixed parameters one. Figures 3 a) and b) show the performance degradation due to mismatch in the statistics of the DCs. For FP $=0$ the DCs probability function is known, while for FP = 1 an uniform probability with the support of the true one is used. When $\mathrm{SH}=5$, a shift $(25 \%)$ of the support of the DCs probability function is considered in the temporal localization of the DCs. If $\mathrm{SH}=0$, no mismatch exists. For a large overlapping between replicas $\left(\bar{\Delta}_{q}=80 \%\right)$, the proposed processor shows to be robust to statistics mismatch. However, for a smaller overlapping, figure $3 \mathrm{~b}$ ) shows some sensitivity to shifts mismatch. In both cases, the assumption of an uniform probability function introduces only a small degradation on the processor.

Regarding the sequential detection of replicas, and for mean overlapping between replicas of $50 \%$, three scenarios are considered: i) small $\mu^{\text {sup }}$ and large $\mu^{\text {inf }}$; ii) the opposite of i); and iii) an intermediate situation, between i) and ii). Table 1 shows the probabilities of detection (PD) and of false alarm (PFA), and the percentage of computational complexity (CC) needed, comparing with the processor that waits for the arrival of all the 10 replicas, for which $\mathrm{PD}=0.8442$ and $\mathrm{PFA}=0.1$. In situation i) the $\mathrm{CC}$ reduces drastically but the PD and the PFA also suffer an important degradation. In this case, detection is performed at the arrival of few replicas. In situation ii), although there is only a small loss of performance, the CC is still reduced to $65.31 \%$.

\section{CONCLUSION}

The optimal processor for stochastic transient signal detection in a multipath environments is, in general, computationally untractable. This paper presents a computationally efficient suboptimal solution, where the multipath parameters are modelled as random variables. A structure for sequential detection of replicas is proposed, avoiding the need to wait for all replicas to make a decision. The proposed solution is robust to the multipath statistics mismatch, thus requiring only mild a-priori information about the channel.

\section{REFERENCES}

[1] I. M. G. Lourtie and G. C. Carter, "Signal detectors for random ocean media," J. Acoust. Soc. Am., vol. 92, no. 3, pp. 14201427, September 1992.

[2] C. He and J. M. F. Moura, "Focused detection via multiresolution analysis," IEEE Transactions on Signal Processing, vol. 46, no. 4, pp. 1094-1104, April 1998.

[3] F. M. Garcia and I. M. G. Lourtie, "Efficiency of real-time Gaussian transient detectors: Comparing the Karhunen-Loève and the wavelet decompositions," in IEEE International Conference on Acoustics, Speech and Signal Processing, Istanbul, Turkey, June 2000.

[4] F. M. Garcia, Detecção Passiva de Sinais Transientes, Ph.D. thesis, Instituto Superior Técnico, September 2000.

[5] K. Fukunaga, Introduction to Statistical Pattern Recognition, Second Edition, Academic Press, 1990. 